\title{
Diffusion-weighted magnetic resonance spectroscopy boosted by simultaneously acquired water reference signals
}

\author{
André Döring ${ }^{1,2}$, Victor Adalid ${ }^{1,2}$, Chris Boesch ${ }^{1}$, and Roland Kreis ${ }^{1}$ \\ ${ }^{1}$ Depts. Radiology and Biomedical Research, University of Bern, Bern, Switzerland \\ ${ }^{2}$ Graduate School for Cellular and Biomedical Sciences, University of Bern, Bern Switzerland
}

Word count: 4776

Running Head: Diffusion-weighted MRS boosted by simultaneously acquired water signals

\section{Corresponding author:}

Prof. Dr. sc. nat. Roland Kreis,

AMSM, University Bern,

Erlachstrasse 9a,

CH-3012 Bern, Switzerland

Tel: +41-31-632 8174

Email: roland.kreis@insel.ch 


\section{Abstract \\ Purpose}

To combine the metabolite-cycling technique with diffusion-weighted ${ }^{1} \mathrm{H}-\mathrm{MR}$ spectroscopy and to use the inherent water reference for compensation of motion-related signal loss for improved estimation of metabolite apparent diffusion coefficients (ADCs).

\section{Methods}

Diffusion-weighted spectra of water and metabolites were acquired simultaneously using metabolitecycling at 3T. The water information was used for signal correction of phase, frequency and eddy currents and compensation of motion-induced signal loss. ADCs were estimated by 2D simultaneous fitting. The quality of ADC restoration was investigated in vitro. Subsequently, the new approach was applied in thirteen subjects for enhanced metabolite ADC estimation in gray matter.

\section{Results}

Metabolite-cycled diffusion ${ }^{1} \mathrm{H}-\mathrm{MRS}$ is suitable to measure metabolite and water ADCs simultaneously. The water reference facilitates signal amplitude restoration compensating for motion-related artefacts. 2D fitting stabilizes the fitting procedure and allows the estimation of ADCs even for low signal-to-noise metabolites. Use of the motion-compensation scheme leads to estimation of smaller ADCs for virtually all metabolites ( $44 \%$ smaller ADC on average), to a reduction of fitting uncertainties for metabolite ADCs in individual subjects and reduced variance over the cohort (45\% smaller standard deviation on average).

\section{Conclusion}

Using the simultaneously acquired water signal as internal reference allows not only for compensation of phase- and frequency-fluctuations but also for signal amplitude restoration and, thus, improved metabolite ADC estimation. Combination with 2D simultaneous fitting promises access to the diffusion properties even for low-signal-to-noise metabolites. The combination of both techniques increases the specificity and sensitivity of estimated metabolite ADC values in the cohort.

\section{Keywords}

MR spectroscopy; diffusion; motion; artefact; quantification; brain; metabolites; signal correction 


\section{Introduction}

The Stejskal-Tanner MR sequence allows the measurement of self-diffusion coefficients of molecules by estimating the signal decay induced by the application of field gradient pulses (1). Due to cellular compartmentation into extra- and intracellular space, spatial restrictions, and viscosity variations the apparent diffusion coefficients (ADCs) observed in vivo differ from diffusion constants measured in vitro in pure liquids. Thus, diffusion-weighted MR spectroscopy (DW-MRS) (2-4) entails information on tissue microstructure (5). Unlike water, metabolite diffusion is mostly restricted to intracellular space (e.g. to neuronal or glial cells $(6,7))$, but the extraction of this particularly valuable information is hindered by different obstacles: (i) the metabolite signals are overwhelmed by the many thousand times stronger water signal (if this is not saturated); (ii) DW-MRS is limited by low SNR especially at high diffusion weighting (high b-values) and (iii) micro- and macro-scale motion disguises as an artificial diffusion contribution distorting the true ADC value. Current DW-MRS methods make use of a combination of water suppression, averaging over many measurements together with cardiac or respiratory gating $(8,9)$. Moreover, linear motion leads to signal phase variation and can be compensated by phasing the signals in postprocessing, which compensates for artificial signal loss from incoherent averaging misinterpreted as faster diffusion $(10,11)$. However, this requires sufficient signal for accurate phase estimation in single acquisitions and sets a limit to the extent of water suppression. Non-linear motion leads to loss in signal amplitude that is not recoverable from single shots, but possibly, as shown below, from a set of high SNR scans.

In this contribution, we suggest an alternative sequence design for DW-MRS using a non-watersuppressed diffusion-weighted MR spectroscopy (nWS-DW-MRS) sequence with metabolite-cycling (MC) and FLAIR cerebrospinal fluid (CSF) suppression, which allows measuring metabolite and water signals simultaneously (12-14). In addition, a novel correction scheme is introduced allowing artefact correction and motion compensation (MoCom) using the co-acquired water signal as inherent reference that enables compensation also for non-linear motion, rendering cardiac and respiratory gating dispensable. Furthermore, a simultaneous 2D spectrum-ADC fitting using the FiTAID toolbox is included to maximize enforcement of prior knowledge constraints $(15,16)$. First, the technique is applied in a phantom with and without motion, showing that the new MoCom scheme leads to improved ADC estimation without triggering. Second, it is demonstrated that the new scheme leads to improved $A D C$ estimation in vivo for a single volunteer and the benefit in applications in medical studies is investigated in a cohort of 13 volunteers. 


\section{Methods}

\section{Data acquisition}

All experiments were conducted on 3T MR scanners (VERIO, TRIO, or PRISMA, Siemens, Erlangen, Germany) using a single proton channel transmit/receive volume coil $\left({ }^{1} \mathrm{H} /{ }^{31} \mathrm{P}\right.$ head coil, RAPID Biomedical, Rimpar, Germany) or multichannel head coils (20 or 32 channels). Based on the Siemens product STEAM sequence, a nWS-DWS sequence, illustrated in Figure 1, was implemented $(12,13,17)$. Pairs of pulsed diffusion field gradients were inserted before and after the mixing time (TM). The crusher gradients in echo time (TE) and TM were adjusted in amplitude, polarity and duration to avoid interfering spurious echoes and minimize eddy currents. An adiabatic MC pulse (14) placed in the TM period of STEAM (17) for up- and downfield frequency-selective magnetization inversion in alternating scans yielded in-phase water, but alternating-phase metabolite signals, noted as $\vec{S}^{\text {up }}$ and $\vec{S}^{\text {down }}$. TE was kept as short as possible to reach high b-values on one side but also to detect metabolites with short $T_{2}$ and with minimal J-coupling evolution on the other. The observed water signal was restricted to the same compartment as the metabolites by CSF suppression based on a water-selective inversion pulse with an inversion time (TI) optimized for CSF signal nulling for the time of the first $90^{\circ}$ pulse. The acquisition parameters for all scans were identical: $T I / T E / T M / T R=1500 / 37 / 150 / 3500 \mathrm{~ms}$. To maximize the achievable b-value, the same diffusion gradient amplitude was applied in all three directions $\left(G=G_{x}=G_{y}=G_{z}\right)$ yielding an effective gradient amplitude $G_{\text {eff }}=\sqrt{3} G$. The b-value was calculated assuming rectangular diffusion gradient pulses and neglecting slice selection and further crusher gradients:

$$
b=\gamma^{2} G_{e f f}^{2} \delta^{2}(\Delta-\delta / 3)
$$

where the diffusion gradient length $\delta=11 \mathrm{~ms}$, the diffusion time $\Delta=168 \mathrm{~ms}$, and a maximal diffusion gradient strength per axis of $35 \mathrm{mT} / \mathrm{m}$ yielded a maximal $b$-value $b_{\max }=5236 \mathrm{~s} / \mathrm{mm}^{2}$. Because of signal loss with increasing b-values, 32 - 128 acquisitions per spectrum were acquired. No triggering was applied.

The sequence was tested in vitro in phantom measurements using a single channel head coil where the scanner software does not automatically phase the single shot spectra such that motion induced phase and signal distortions could be examined. Rotational motion around the vertical axis was realized by placing a spherical phantom ("braino" phantom from GE Medical systems) on a wooden rack in the head coil. Rotation with an angle of $\approx 10^{\circ}$ and a frequency of $\approx 0.5 \mathrm{~Hz}$ was realized by two ropes attached to the phantom. During the sequence, the phantom was kept static for $15 \%$ of the acquisitions to guarantee a reproducible non-distorted reference. The phantom sphere was filled with 
an aqueous solution of the brain metabolites $\mathrm{ml}$ (myo-inositol, $7.5 \mathrm{mM}$ ), NAA ( $\mathrm{N}$-acetyl aspartate, $12.5 \mathrm{mM}$ ), Glu (glutamate, $12.5 \mathrm{mM}$ ), $\mathrm{Cr}$ (creatine, $10.0 \mathrm{mM}$ ), Lac (lactate, $5.0 \mathrm{mM}$ ) and Cho (choline, $3.0 \mathrm{mM}$ ). For sensitivity reasons, the in vivo spectra were acquired using multi-channel head coils with inherent phase-correction through the manufacturer's coil combination routine. Thirteen healthy volunteers (age: $31.4 \pm 10.0 \mathrm{yr}$; range $20-57$; 4 women/9 men) were investigated with a region of interest (ROI) placed midline in occipital cortex dominated by gray matter (GM). The ROI size was maximized for each volunteer taking care to prevent contamination by subcutaneous fat signal (voxel size: $\left.\mathrm{V}_{\min }=12.9 \mathrm{~mL} ; \mathrm{V}_{\max }=21.8 \mathrm{~mL} ; \mathrm{V}_{\emptyset}=(16.5 \pm 2.9) \mathrm{mL}\right)$. The flip angle was optimized within the ROI using a $B_{1}$ map. Shimming was performed using automatic second order shimming yielding a mean Voigt linewidth of $(7.2 \pm 0.5) \mathrm{Hz}$ for the water signal. Single acquisitions were stored without averaging.

\section{Motion-compensation scheme}

Signal processing, in particular a fully automated motion-compensation algorithm, was implemented in MatLab ${ }^{\circledR}$ (version 8.5.0 R2015a, Mathworks, Natick, Massachusetts, U.S.A.). The correction scheme comprises two principal stages applied to all acquisitions acquired at an individual b-value to account for motion induced artefacts and signal loss in the metabolite and water spectra. The first stage accounts for inter-acquisition distortions (Figure S1 in the supplementary materials) using the inherent water reference signal for the well-established correction of phase-fluctuations (PhCor), frequencydrift (FrCor) and eddy-current distortions (EcCor). Phase shifts in single acquisitions, related to linear motion, were corrected to prevent artificial signal loss from incoherent averaging. The complex acquisition vectors are rotated in the time domain around a fixed angle maximizing the real and nulling the imaginary part of the first data points of all acquisitions $(10,11)$. A frequency drift is corrected by applying a linear phase shift in the time domain maximizing the product of each acquisition with a reference scan (18). A time varying signal-phase introduced by eddy currents is corrected in the time domain by decomposing the up- $\vec{S}^{\text {down }}$ and downfield $\vec{S}^{u p}$ inverted acquisitions pairwise into the metabolite $\vec{S}_{\text {metab }}$ and water $\vec{S}_{\text {water }}$ contributions.

$$
\begin{aligned}
& \vec{S}_{\text {metab }}=\vec{S}^{\text {down }}-\vec{S}^{\text {up }} \\
& \vec{S}_{\text {water }}=\vec{S}^{\text {down }}+\vec{S}^{\text {up }}
\end{aligned}
$$

The spurious time-dependent phase, generated by eddy currents, is inherent in the water signal and corrected in the metabolite and water signal by multiplication with the inverted phase vector (19). Finally, the up- and downfield inverted single acquisitions are regenerated. 
The second stage of water referencing (presented in Figure 2) is novel. It quantifies and restores signal quality and potential loss of signal amplitude in individual acquisitions.

The correction parameters are derived from the dominating water signal contribution in individual acquisitions. Since the correction scheme is highly sensitive to wrongly estimated correction parameters, bad quality water signals can compromise the overall correction scheme and lead to distorted metabolite spectra. Thus, outlier criteria are defined to ensure a water signal quality sufficient for derivation of correction parameters. The correction parameters are determined by fitting of the time domain free induction decay (FID) to a complex Voigt decay:

$$
v_{j}(t)=A_{j} \cdot e^{-\pi L_{j} t} \cdot e^{-\frac{\left(\pi G_{j} t\right)^{2}}{4 \ln 2}} e^{-i\left(2 \pi f_{j} t+\Phi_{\mathrm{j}}\right)}
$$

The fitting parameters are the signal amplitude $A_{j}$, the Lorentzian linewidth $L_{j}$, the Gaussian linewidth $G_{j}$, the offset frequency $f_{j}$ and the signal phase $\Phi_{j}$ of the single acquisition $j$ at any specific b-value with associated fitting uncertainties $\Delta A_{j}, \Delta L_{j}, \Delta G_{j}, \Delta f_{j}$ and $\Delta \Phi_{j}$ given by the $95 \%$ confidence intervals (CI) $(15,20)$. The two linewidths are combined into an estimate of the overall Voigt width (21)

$$
V_{j}=0.5346 \cdot \mathrm{L}_{\mathrm{j}}+\sqrt{0.2166 \cdot L_{j}^{2}+G_{j}^{2}}
$$

The fit uncertainty $\Delta V_{j}$ of the Voigt linewidth is estimated by error propagation

$$
\begin{aligned}
\Delta \mathrm{V}_{j} & =\left|\frac{\partial V}{\partial L}\right| \Delta L+\left|\frac{\partial V}{\partial G}\right| \Delta G \\
& =\left(0.5346+\frac{0.2166 \cdot L_{j}}{\sqrt{0.2166 \cdot L_{j}^{2}+G_{j}^{2}}}\right) \cdot \Delta L_{j}+\left(\frac{G_{j}}{\sqrt{0.2166 \cdot L_{j}^{2}+G_{j}^{2}}}\right) \cdot \Delta G_{j}
\end{aligned}
$$

Single acquisitions of poor quality were excluded if relative fitting uncertainties of the estimated amplitude or Voigt width were $>5 \%$

$$
\begin{aligned}
& \frac{\Delta V_{j}}{V_{j}}>0.05 \quad \text { or } \\
& \frac{\Delta A_{j}}{A_{j}}>0.05
\end{aligned}
$$

To prevent contributions from outliers that pass these quality criteria, but stand out in comparison to the other equivalent acquisitions, the standard deviations over all acquisitions per b-value were calculated for areas $\left(\sigma_{A}\right)$ and Voigt linewidths $\left(\sigma_{V}\right)$. These outliers were defined separately for up- and downfield inverted acquisitions and discarded when 


$$
\begin{aligned}
& A_{j}^{u p / \text { down }}<\overline{\mathrm{A}}^{\text {up } / \text { down }}-2 \sigma_{A}^{u p / \text { down }} \text { or } \\
& V_{j}^{\text {up } / \text { down }}>\bar{V}^{\text {up } / \text { down }}+2 \sigma_{V}^{u p / \text { down }}
\end{aligned}
$$

where $\overline{\mathrm{A}}$ and $\overline{\mathrm{V}}$ denote the average values of the area and linewidth, respectively, at any individual bvalue.

The next step deals with restoration of corrupted signals and compensation of motion-related signal loss. To this end, the median value of an arbitrarily sized top quantile of all acquisitions at an individual b-value was used to define a reference level $A_{r e f}^{u p / d o w n}$ - representing an estimate for the signal level for acquisitions that are not compromised by motion. This was again done separately for up- and downfield inverted acquisitions. To examine the effect, of the choice of the quantile level, evaluations with $25 \%$ and $15 \%$ were compared for the rotating phantom data (where $15 \%$ was known to match the number of uncompromised acquisitions). For the in vivo measurements, the top quantile level was set to $15 \%$.

This reference amplitude was then used for the calculation of scaling factors

$$
\rho_{j}^{u p / \text { down }}=A_{r e f}^{u p / \text { down }} / A_{j}^{u p / \text { down }}
$$

that restore the motion-induced amplitudes to the reference levels

$$
\vec{S}_{\text {restored,j }}^{u p / \text { down }}=\rho_{j}^{u p / \text { down }} \cdot \vec{S}_{j}^{u p / d o w n}
$$

To prevent a substantial decrease in SNR, weighted averaging similar to the optimized summation of data from multichannel RF coils was employed (22-24). Using SNR squared as weighting factor, where SNR is inversely proportional to the scaling factors in our case, yields for the restored summed signals

$$
\vec{S}_{\text {restored }}^{\text {up } / \text { down }}=\sum_{j} \frac{\vec{S}_{j}^{\text {up } / \text { down }} / \rho_{j}^{\text {up } / \text { down }}}{\sum_{k}\left(1 / \rho_{k}^{\text {up } / \text { down }}\right)^{2}} .
$$

Finally, equation [2] yields the summed metabolite $\vec{S}_{\text {metab }}^{\text {restored }}$ and water $\vec{S}_{\text {water }}^{\text {restored }}$ signals for each bvalue.

\section{Fitting procedure}

Spectra were fitted with prior-knowledge enhanced linear combination models using the Fitting Tool for Arrays of Interrelated Datasets (FiTAID) (15). As recently described (16), this tool has been 
extended to support simultaneous modeling of the spectral and diffusion domains, where the diffusion effect is represented by a mono-exponential signal attenuation:

$$
A(b)=\mathrm{A}_{0} e^{-b A D C} .
$$

$b$ is the $b$-value as defined above, $A(b)$ the area of a specific metabolite at a specific $b$-value and $A_{0}$ the theoretical metabolite area without diffusion weighting. For the in vivo data, the basis set comprised 19 metabolites (Asp: aspartate; Cr; Etn: ethanolamine; GABA: $\gamma$-aminobutyric acid; Glc: glucose; Gln: glutamine; Glu; Gly: glycine; GPC: glycerophosphorylcholine; GSH: glutathione; Lac; ml; NAA; NAAG: N-acetylaspartylglutamate; PCho: phosphocholine; PCr: phosphocreatine; PE: phosphorylethanolamine; sl: scyllo-inositol; Tau: taurine;) with spectra simulated using $\operatorname{VESPA}(25,26)$ assuming ideal RF pulses. The basis also included an experimentally measured macromolecular (MM) baseline as obtained from metabolite-nulled PRESS spectra at $35 \mathrm{~ms}$ TE. The signal from residual water was included as a single Voigt contribution at $4.7 \mathrm{ppm}$. The fitting range in the frequency domain was restricted from 0 to $4.1 \mathrm{ppm}$. Resonances were modeled with Voigt lineshapes. The Lorentz broadening was fixed to $2.2 \mathrm{~Hz}$ (corresponding to a $\mathrm{T}_{2} \approx 145 \mathrm{~ms}$ ), except for NAA with $1.1 \mathrm{~Hz}$ width and the MM baseline, where the Lorentzian decay is already included in the experimental pattern. A common Gauss broadening was included, which represents the field inhomogeneity contribution to the linewidth. All lines were modeled with equal zero-order phase, frequency shift and Gaussian broadening at each b-value, but these parameters were allowed to vary from one b-value to the next. FiTAID was also used to determine Cramér-Rao Lower Bounds (CRLB), i.e. estimates of the minimal fitting error for all estimated parameters.

Two fitting approaches were used, one where the spectral and diffusion dimensions were modeled together, and a another with independent fitting of all spectra. In the former, the (i) simultaneous fitting approach, the metabolite ADCs were determined directly by simultaneous 2D fitting in FiTAID. The ADCs were constraint to positive values for all metabolites but not the macromolecules. Due to the very slow MM diffusion, noise and baseline fluctuation can lead to negative estimates of this ADC. In order for a cohort average to represent a good estimate of the true MM ADC, individual estimates were thus not restrained to positive values. In the latter, the (ii) sequential fitting approach, metabolite areas were determined by sequential 1D fitting of the spectra at each b-value, followed by modeling of the diffusion decay in MatLab ${ }^{\circledR}$. Metabolite ADCs were estimated using a weighted fitting routine, where the inverse of the respective CRLB was used as weight. In agreement with the simultaneous fitting approach, the ADCs were modeled by a mono-exponential decay function Eq. [11]. In addition to the $A D C$ values, the MatLab ${ }^{\circledR}$ fit yields $\mathrm{A}_{0}$ and the corresponding fit uncertainties $\triangle A D C$ and $\Delta A_{0}$, which - in contrast to the CRLB - are related to the residuals and determine a $95 \% \mathrm{Cl}$ of the 
estimated parameters (20). To eliminate outliers, the fit was performed in a two-step procedure. First, all area values were used to determine a first estimate of $\mathrm{ADC}$ and $\mathrm{A}_{0}$, and their $95 \% \mathrm{Cl}$. For the second fit, only experimental areas $A(b)$ lying within this interval were used. To be in concordance with the constraints on positive ADCs in the simultaneous fitting approach negative ADCs, except for the MMs, were discarded.

To minimize bias in the cohort average for ADC values, probable outliers were eliminated from the dataset if the uncertainties of an estimated individual ADC was much larger than the median uncertainty for this value over the cohort data (in absolute, not relative terms (27)). The uncertainties correspond to the CRLBs in case of simultaneous fitting and to the $\mathrm{Cls}$ for sequential fitting. Individual metabolite ADCs were thus excluded from the cohort evaluation if

$$
\mathrm{ADC}_{\mathrm{CRLB}_{i}^{\text {metab }}}>2 \cdot \text { median }\left(\mathrm{ADC}_{\mathrm{CRLB}_{i}^{\text {metab }}}\right) \text { for simultaneous }
$$

fitting (i)

$$
\Delta \mathrm{ADC}_{i}^{\text {metab }}>2 \cdot \text { median }\left(\Delta \mathrm{ADC}_{i}^{\text {metab }}\right) \text { for sequential fitting (ii) }
$$

where $\mathrm{ADC}_{-} \mathrm{CRLB}_{i}^{\text {metab }}$ and $\triangle \mathrm{ADC}_{i}^{\text {metab }}$ denote the subject and metabolite specific uncertainties of the estimated $\mathrm{ADCs}$, and median $\left(\mathrm{ADC}_{-} \mathrm{CRLB}_{i}^{\text {metab }}\right)$ and median $\left(\triangle \mathrm{ADC}_{i}^{\text {metab }}\right)$ the corresponding median values for the cohort.

\section{Results}

\section{In vitro testing}

\section{Stationary phantom}

Experimental tests on a stationary phantom showed that the proposed motion-compensation algorithm does not influence the estimated $A D C$ values if there is no gross motion-related signal reduction present. However, the evaluation showed that the previously proposed phase, frequency and eddy current correction of individual acquisitions is indeed beneficial also in a phantom that is not moved intentionally, but only suffers from general instabilities including gradient-pulse-induced vibrations, amplifier heating and signal inhomogeneities from eddy currents. Detailed results on defining the ground truth (GT) are documented in the supplemental Figure S2.

\section{Moving phantom}

Figure 3 illustrates sample spectra for simultaneous acquisition of DW metabolite and water spectra and visualizes the success of the proposed MoCom scheme in a rotating phantom, where GT is known from the data of the stationary phantom as described above. The influence of rotational motion on 
DW spectra, the derived ADC values and the benefit from the MoCom algorithm is visualized in Figure 3B by difference spectra with respect to the GT data displayed in Figure 3A. Without any correction, a substantial general loss of signal of $>50 \%$ is observed, reflected in very large difference spectra for all b-values. This signal loss is somewhat smaller with application of PhCor. However, PhCor alone is not sufficient to restore the GT signal amplitudes. When applying MoCom in addition to PhCor, the motion-related signal loss can essentially be reversed, which corresponds to very small spectral features in the difference spectra. The resulting metabolite ADCs obtained from 2D fitting are summarized in Figure 4. Simple averaging of uncorrected acquisitions yields estimated metabolite ADCs that are more than double the GT values. In addition, the inherent differences in the GT ADCs between metabolites are not conserved. With PhCor alone, the average bias is reduced to $\approx 60 \%$ and the relative differences between metabolites are almost restored. With PhCor plus MoCom the bias for ADC estimation is reduced drastically. For a reference level of $15 \%$, i.e. only using truly unaffected acquisitions, the bias is a mere $2 \%$ on average, while a larger reference level of $25 \%$ incurs a remaining bias of $\approx 13 \%$. For both cases, the inherent differences in ADCs between metabolites are retained.

\section{In vivo testing in human subjects}

Figure 5 shows an exemplary set of DW spectra for the single subject that showed the largest signal fluctuations in the cohort. The effects of MoCom restoration for water and metabolite spectra are presented at nine b-values as a comparison between the spectra without MoCom in the upper and with MoCom (reference level of 15\%) in the middle row (PhCor applied for both sets). Careful comparison between the sets clearly reveals differences induced by the MoCom scheme, which mainly pertain to signal intensity while the noise level remains similar. Difference spectra (shown in the lowest panel) illustrate the motion-induced signal loss best. Here, it is evident that the extent of the differences depends on b-value. The motion-induced signal loss that is restored by MoCom increases up to $\mathrm{b}=1710 \mathrm{~s} / \mathrm{mm}^{2}$ for water and metabolites. For higher $\mathrm{b}$-values the absolute signal loss for water largely diminishes while for the metabolites it remains about the same. However, in relative terms, motion leads to ever increasing amplitude effects with increasing diffusion weighting. At the highest $b$-value of $\approx 5000 \mathrm{~s} / \mathrm{mm}^{2}$, the water signal has decayed by $>95 \%$ causing $42 \%$ of acquisitions to be discarded by the chosen quality filter, though the remaining acquisitions were still sufficient for application of the MoCom scheme. Seen from the cohort data, typically, less than $10 \%$ of acquisitions have to be rejected and a $20-40 \%$ rejection rate is reached for single cases only.

The deduced ADCs and their CRLBs estimated with 2D fitting are collected in Figure 6 for the same subject as in Figure 5. As expected, MoCom leads to a signal increase especially at higher diffusion weightings. For water, the estimated $A D C$ reduces by $18 \%$ from $9.5 \cdot 10^{-4} \mathrm{~mm}^{2} / \mathrm{s}$ to 7.8 . 
$10^{-4} \mathrm{~mm}^{2} / \mathrm{s}$. The calculated CRLBs are on the order of $10^{-8} \mathrm{~mm}^{2} / \mathrm{s}$, four orders of magnitude lower than the diffusion constants themselves. However, these values were drawn from a mono-exponential fit restricted to data obtained with $\mathrm{b} \leq 1710 \mathrm{~s} / \mathrm{mm}^{2}$. For higher $\mathrm{b}$ values, the decay deviated markedly from a single exponential - both with and without MoCom. The metabolite diffusion exhibits a mono-exponential signal decay up to the highest $b$-value of $b=5236 \mathrm{~s} / \mathrm{mm}^{2}$ (exemplarily documented in Figure $\mathrm{S} 3$ in the supplementary materials using results for some of the metabolites from the consecutive spectral-diffusion domain fit) justifying use of all data for mono-exponential diffusion modeling. As shown in Figure 6, all metabolite ADCs (except Tau) are lower after MoCom (by $53 \%$ on average). It is noteworthy that also the absolute CRLBs of the metabolite ADCs are lowered by $17 \%$ on average.

The overall results for the whole cohort as obtained with and without MoCom and based on the simultaneous fit method are presented in the boxplot in the upper part of Figure 7. The effect of MoCom on the evaluation of the ADCs is given in the lower part of this figure. The mean ADC of water without MoCom is $8.2 \cdot 10^{-4} \mathrm{~mm}^{2} / \mathrm{s}$ with a SD over the cohort of $0.5 \cdot 10^{-4} \mathrm{~mm}^{2} / \mathrm{s}$. The ADC estimated with MoCom shows a reduced mean value of $(7.7 \pm 0.4) \cdot 10^{-4} \mathrm{~mm}^{2} / \mathrm{s}$, i.e. a reduction by $6 \%$.

The effect of MoCom on the metabolite ADCs is stronger, with an average reduction in mean and median ADC of $44 \%$ and $38 \%$, respectively. Besides the direct effect on the ADCs there is a remarkable improvement of cohort variance with the SD reduced by $45 \%$ and also an increase in the number of valid estimates by $11 \%$. Considering the effect for individual metabolites, MoCom leads in almost all cases to lowering of the mean, median and SD values.

Overall very similar results were obtained when using sequential fitting of the spectral and diffusion domains. They are presented in Figure $\mathrm{S} 4$ in the online supplement. 


\section{Discussion}

Separately or simultaneously acquired water reference acquisitions have been used previously in MRS for restoration of phase, frequency or lineshape distortions or for quantification purposes using water as intensity reference $(10,11,18,19)$. Simultaneously recorded water data has also been used to exclude motion-related corrupted acquisitions (28). Here, water-referencing is extended to novel use. We demonstrate that, based on an ensemble of equivalent recordings, a simultaneously recorded water reference can be used to restore loss of signal amplitude in single acquisitions. This approach was found to be very valuable in the case of DW-MRS, where signal intensity in single acquisitions can be affected drastically by non-linear bulk motion and where this signal loss is equal for the high SNR water peak and the low SNR metabolite signals. Hence, knowledge about the extent of the signal deficit can be transferred from water to the metabolites. The validity of this amplitude referencing has been demonstrated in vitro on a rotating phantom, where ADC values close to ground truth can be determined even if a clear majority of scans is strongly distorted by motion.

Given that the extent of signal loss in diffusion scans scales with the strength of the diffusion weighting, motion-related signal drop normally leads to misinterpretation of the bulk motion as faster diffusion $(11,29)$. As shown in the phantom and the in vivo data from human brain gray matter, the proposed water-amplitude referencing in the form of the described MoCom scheme leads to smaller estimated ADC values for metabolites and water that are most likely closer to true ADCs even though the recordings did not include any triggering to minimize motion effects. Lower CRLB and reduced inter-subject variance for the determined metabolite ADC values confirm that the novel postprocessing tool improves the estimation quality for the diffusion characteristics of metabolites; in particular those represented with low signal intensities in brain spectra obtained in vivo in human subjects.

The validity of the MoCom scheme clearly relies on the assumption that a portion of the acquisitions that is equal or larger than the percentile used to calculate the reference level is essentially unaffected by motion. Furthermore, the corrected metabolite scans are only closer to the true intensities, if the uncertainty in the determination of the water signal area related to spectral noise is smaller than the motion-related signal drop.

In addition, the method relies on the assumption that there is no mechanism by which single acquisitions could show a substantial artificial intensity increase (i.e. in DW MRS, water signal can only be lost, not gained by artefacts). If fewer acquisitions than assigned to reference scans are unaffected by motion, the correction will only be partial (as seen in the example of the rotating phantom when the top $25^{\text {th }}$ percentile was used for reference definition, while only $15 \%$ of the scans had been recorded at rest). To minimize this danger, one could in principle use the single acquisition with the 
maximum signal to define the reference level. However, that might lead to too high a reference level for single $b$-values because of noise-related amplitude over-estimation (relevant at high b-values with strongly reduced water signal) or also because partial volume effects or incomplete CSF suppression in single acquisitions might yield overestimated amplitudes in single shots. Hence, the top $15^{\text {th }}$ percentile of all acquisitions was used in the current implementation as a compromise that seemed to work well in vivo where a minimum of 32 to 128 acquisitions per b-value were recorded. How well the reference level is defined based on the top $15^{\text {th }}$ percentile can also be visually appreciated from a plot of estimated water areas. Supplemental Figure S5 documents how the clearly defined maximum attainable signal transfers to the reference level for two cases, one with large and one with little motion-related signal fluctuation.

At very high b-values, the proposed scheme could become problematic. Since water diffuses more rapidly than metabolites, one can arrive at a scenario where the diffusion-weighted water signal has similar or even lower SNR than the metabolite spectrum. Obviously, at that stage, one would not try to base a correction on the water signal. But already at b-values that lead to a variance in the estimated water signal that approaches the range of the expected amplitude corrections, the current MoCom scheme would not be suitable because it might lead to underestimation of ADC values for water and the metabolites. However, up to the currently used maximum b-values (and number of acquisitions and top percentile used) such a bias is very unlikely.

It should be noted that this correction scheme works independent of the nature of the diffusionrelated water signal decay. Because data from each b-value is considered separately the water signal decay can be faster or slower than that of the metabolites or the decay can be non-Gaussian. The only condition is that there is enough water signal left at each b-value to define a meaningful reference level within the selected reference ensemble. Furthermore, in the current implementation of the acquisition sequence, inversion-based nulling of the CSF water signal was included to guarantee equivalent compartments for the origin of water and metabolite signals, which is crucial if the ADC values of water and metabolites are compared and interpreted. It is expected that the MoCom intensity correction is also more reliable with CSF-nulling because compartments with the same motion characteristics are implicated for metabolites and water, but this has not been investigated. It should be stressed that the accuracy and stability of CSF suppression is probably not critical because a) it concerns modest inaccuracy for a minor contributor to the overall water signal; and b) at high bvalues where MoCom is critical the CSF signal does not contribute much anyway because of much faster diffusion constants for CSF than parenchymal water.

Most of the results have been obtained using simultaneous spectral and diffusion modeling $(15,16)$. However, similar results have also been gained with separate spectral and diffusion fitting. 
Simultaneous fitting has been shown to be superior before, which was based on stabilization of the spectral fit by additional data dimension $(15,30-32)$. However - in the present implementation in FiTAID - it has the disadvantage that it does not allow for proper consideration of unequal noise levels in the spectral series if spectra at different b-values have been recorded with unequal numbers of acquisitions. This can be accommodated for in separate modeling of spectra and diffusion data, where it is also easy to consider a non-mono-exponential signal decay.

It should be noted that the simultaneously acquired water reference - beside the novel use for intensity correction - also proved to be very useful for the more conventional signal restoration steps. In particular, it allowed for phase- and frequency-alignment as well as exclusion of single acquisitions of very low quality leading also to improved line widths, signal shape, and thus spectral resolution upon signal averaging $(10,11,18,19)$. Particularly signal phasing was found to be crucial not only for the in vivo but also the in vitro situation, probably because table vibrations induced by large diffusion gradient pulses interfered with coherent averaging otherwise.

The proposed motion compensation scheme may be considered complementary to standard MRnavigator or optical motion tracking methods. In particular, optical real-time motion tracking may well be used in addition to the proposed scheme. It may allow to prevent signal corruption due to gross head motion, though small local motion of brain tissue or motion that is too fast to be corrected in real-time could then still be compensated by the proposed MoCom scheme.

In the current implementation, the definition of the reference level and hence scaling factors for individual acquisitions is possible because the water resonance is not suppressed. One may wonder whether a similar approach might also work in case of water-suppressed data. Given the SNR of the highest metabolite peaks in single acquisitions in frequency domain (SNR $<2$ for highest $b$ values and the ROI size as used in this study), a correction similar to the one proposed using the water signal is not possible in a straightforward manner. This SNR may be sufficient for phasing or frequency shifts (as shown in (33)), but not for setting a reference level needed for intensity correction. Obviously, this might work for weakly water-suppressed spectra, but then the water suppression efficiency would have to be extremely consistent, which is unlikely in case of motion and field drifts. Instead, in an exploratory fashion we looked into fitting individual acquisitions (with the water signal removed) by a pattern of the overall fitted spectrum. This confirmed that for the present acquisition parameters, such an approach would not be good enough to define scaling factors reliably and lead to additional bias. However, noise-related area fluctuations for water-suppressed single acquisition spectra appeared to be only around $10-15 \%$ for our largest b-values; hence, with moderately improved SNR (e.g. from 7T data or from high field animal scanners or with localization schemes without SNR penalty as in STEAM) a similar MoCom scheme might be feasible also for DW MRS with water presaturation. 
As alternative approach for mitigation of motion-related signal drop, it is possible to resort to assuming that macromolecular signals do not diffuse or at least diffuse much slower (and/or at a predefined rate) than the metabolites. In that case, the sum spectra (including the motion-corrupted scans) can be scaled such that the macromolecular peak is at a constant or expected level for each b-value. Such a correction scheme is similar to what had been suggested for investigations of metabolite diffusion in skeletal muscle, where the lipid signals had been used as reference (12). The use of the macromolecular peak at $0.9 \mathrm{ppm}$ for single shot outlier rejection (34) has been used for animal studies, but relies on much better SNR than available in our human studies.

As mentioned, simultaneous modeling with FiTAID is restricted to mono-exponential diffusion-related signal decay. For the water signal, this is not guaranteed if strong b-values are used. Thus, the b-value range for water fitting has to be restricted appropriately. For the metabolites on the other hand, monoexponential decay was found to prevail for the range of $b$-values used in this study. This was verified by sequential fitting as documented for a single subject in the supplemental figure (Fig. S3). For none of the metabolites, a systematic deviation from the monoexponential representation was found. For even larger b-values, this will have to be reconsidered. It should be noted that the influence of motionrelated signal decay might actually be mistaken for a bi-exponential diffusion decay, which can largely be prevented by the proposed MoCom method.

Due to disparate measurement conditions ( $b_{\max }, T E$ and $\Delta$ ) the obtained $A D C$ values cannot be unreservedly compared to other studies in the literature. However, a good agreement of the uncorrected metabolite ADC values of NAA (median: $(1.5 \pm 0.2) \cdot 10^{-4} \mathrm{~mm}^{2} / \mathrm{s}$ ), tCho (mean of median of PCho and GPC: $\left.(1.2 \pm 0.7) \cdot 10^{-4} \mathrm{~mm}^{2} / \mathrm{s}\right)$ and Glu (median: $\left.(1.7 \pm 0.5) \cdot 10^{-4} \mathrm{~mm}^{2} / \mathrm{s}\right)$ with respect to the GM values reported in Ref. (35) is found. Since, the values presented in Ref. (35) are obtained without cardiac or respiratory gating it is reasonable that our ADC values obtained after motion compensation are lower than those reported in the literature. The $A D C$ of $t C r$ is hard to compare because in the present study spectra were fitted with separate $\mathrm{Cr}$ and $\mathrm{PCr}$ patterns. The resulting $A D C$ values for these separate creatine entities, and probably also for minor spectral contributions like GABA or Etn, should be taken with a grain of salt at this point, given the limited SNR in single spectra obtained at $3 \mathrm{~T}$, though the values appear fairly consistent over the cohort for several of these components. Further, it is noteworthy that the diffusion time of $168 \mathrm{~ms}$ is longer and the echo time of $37 \mathrm{~ms}$ is shorter than those usually found in literature. Hence, the estimated metabolite ADCs in our study are more affected by restricted diffusion and possibly inaccurate representation of the macromolecular background. In Ref. (36), longer diffusion times are shown to yield reduced ADC values which could also add to the observed lower motion compensated ADCs found in our study in comparison to Refs. (37-40). 


\section{Conclusion}

The estimation of metabolite ADCs is easily compromised by motion artefacts. It was shown that the proposed motion compensation scheme combined with 2D simultaneous fitting can mitigate this problem. The novel correction scheme partially restores motion compromised diffusion spectra on the basis of the simultaneously acquired water reference signal and yields ADC values closer to the true values. Application of 2D simultaneous fitting improves the fitting stability and accuracy and, thus, allows estimation of ADCs also for low SNR metabolites, not reliably detectable by 1D sequential fitting. The application in cohort studies increases the specificity (more valid estimates) and sensitivity (reduced SD). Combination with real time motion detection may further improve the ADC estimation.

\section{Acknowledgement}

This work is supported by the Swiss National Science Foundation (SNSF \#320030-156952 and \#320030175984). 


\section{References}

1. Stejskal EO, Tanner JE. Spin Diffusion Measurements: Spin Echoes in the Presence of a TimeDependent Field Gradient. J Chem Phys 1965; 42:288-292.

2. Nicolay K, Braun KPJ, De Graaf RA, Dijkhuizen RM, Kruiskamp MJ. Diffusion NMR spectroscopy. NMR Biomed 2001; 14:94-111.

3. Ronen I, Valette J. Diffusion-Weighted Magnetic Resonance Spectroscopy. eMagRes 2015; 4:733750.

4. Cao P, Wu EX. In vivo diffusion MRS investigation of non-water molecules in biological tissues. NMR Biomed 2017; 30:e3481.

5. Bihan D Le. Molecular diffusion, tissue microdynamics and microstructure. NMR Biomed 1995; 8:375-386.

6. Nordengen K, Heuser C, Rinholm JE, Matalon R, Gundersen V. Localisation of N-acetylaspartate in oligodendrocytes/myelin. Brain Struct Funct 2015; 220:899-917.

7. Brand A, Richter-Landsberg C, Leibfritz D. Multinuclear NMR Studies on the Energy Metabolism of Glial and Neuronal Cells. Dev Neurosci 1993; 15:289-298.

8. Najac C, Branzoli F, Ronen I, Valette J. Brain intracellular metabolites are freely diffusing along cell fibers in grey and white matter, as measured by diffusion-weighted MR spectroscopy in the human brain at 7 T. Brain Struct Funct 2016; 221:1245-1254.

9. Upadhyay J, Hallock K, Erb K, Kim D, Ronen I. Diffusion properties of NAA in human corpus callosum as studied with diffusion tensor spectroscopy. Magn Reson Med 2007; 58:1045-1053.

10. Zhu G, Gheorghiu D, Allen PS. Motional degradation of metabolite signal strengths when using STEAM: A correction method. NMR Biomed 1992; 5:209-211.

11. Posse S, Cuenod CA, Le Bihan D. Human brain: proton diffusion MR spectroscopy. Radiology 1993; 188:719-725.

12. Brandejsky V, Boesch C, Kreis R. Proton diffusion tensor spectroscopy of metabolites in human muscle in vivo. Magn Reson Med 2014; 73:481-487.

13. Dreher W, Leibfritz D. New method for the simultaneous detection of metabolites and water in localized in vivo ${ }^{1} \mathrm{H}$ nuclear magnetic resonance spectroscopy. Magn Reson Med 2005; 54:190-195.

14. Hwang TL, van Zijl PC, Garwood M. Asymmetric adiabatic pulses for NH selection. J Magn Reson 1999; 138:173-177. 
15. Chong DGQ, Kreis R, Bolliger CS, Boesch C, Slotboom J. Two-dimensional linear-combination model fitting of magnetic resonance spectra to define the macromolecule baseline using FiTAID, a Fitting Tool for Arrays of Interrelated Datasets. MAGMA 2011; 24:147-164.

16. Adalid V, Döring A, Kyathanahally SP, Bolliger CS, Boesch C, Kreis R. Fitting interrelated datasets: metabolite diffusion and general lineshapes. MAGMA 2017:1-20.

17. MacMillan EL, Kreis R, Fuchs A, Versluis MJ, Boesch C, Boesiger P, Henning A. New Technique for Metabolite Cycled Non-Water-Suppressed Proton Spectroscopy in the Human Brain at 7T. Proc Int Soc Magn Reson Med 2011; 19:1412.

18. Helms G, Piringer A. Restoration of motion-related signal loss and line-shape deterioration of proton MR spectra using the residual water as intrinsic reference. Magn Reson Med 2001; 46:395-400.

19. Klose U. In vivo proton spectroscopy in presence of eddy currents. Magn Reson Med 1990; 14:2630.

20. Lane TP, DuMouchel WH. Simultaneous Confidence Intervals in Multiple Regression. Am Stat 1994; 48:315-321.

21. Olivero JJ, Longbothum RL. Empirical fits to the Voigt line width: A brief review. J Quant Spectrosc Radiat Transf 1977; 17:233-236.

22. Dong Z, Peterson B. The rapid and automatic combination of proton MRSI data using multi-channel coils without water suppression. Magn Reson Imaging 2007; 25:1148-1154.

23. Hall EL, Stephenson MC, Price D, Morris PG. Methodology for improved detection of low concentration metabolites in MRS: Optimised combination of signals from multi-element coil arrays. Neuroimage 2014; 86:35-42.

24. Miller JJ, Cochlin L, Clarke K, Tyler DJ. Weighted averaging in spectroscopic studies improves statistical power. Magn Reson Med 2017; 78:2082-2094.

25. Soher B, Semanchuk P, Todd D, Young K. Vespa: Versatile Simulation, Pulses and Analysis for MR Spectroscopy. 2017. [http://scion.duhs.duke.edu/vespa/]

26. Soher BJ, Semanchuk P, Todd S, Steinberg J, Young K. VeSPA: Integrated applications for RF pulse design, spectral simulation and MRS data analysis. In Proceedings of the 19th AnnualMeeting of ISMRM, Montréal, Québec, Canada, 2011:1410.

27. Kreis R. The trouble with quality filtering based on relative Cramér-Rao lower bounds. Magn Reson Med 2016; 75:15-18. 
28. MacMillan EL, Bortolotti M, Boss A, Boesch C, Kreis R. Metabolite Cycled Non-Water-Suppressed Spectroscopy Offers Increased Spectral Quality In Cases of Physiologic and Subject Motion. Proc Int Soc Magn Reson Med 2011; 19:2996.

29. Chenevert TL, Brunberg JA, Pipe JG. Anisotropic diffusion in human white matter: demonstration with MR techniques in vivo. Radiology 1990; 177:401-405.

30. Van Ormondt D, De Beer R, Mariën AJ., Den Hollander J., Luyten P., Vermeulen JWA. 2D approach to quantitation of inversion-recovery data. J Magn Reson 1990; 88:652-659.

31. de Beer R, van Ormondt D, Pijnappel WWF. Quantification of 1-D and 2-D magnetic resonance time domain signals. Pure Appl Chem 1992; 64:815-823.

32. Schulte RF, Boesiger P. ProFit: two-dimensional prior-knowledge fitting of J-resolved spectra. NMR Biomed 2006; 19:255-263.

33. Deelchand DK, Auerbach EJ, Marjańska M. Apparent diffusion coefficients of the five major metabolites measured in the human brain in vivo at 3T. Magn Reson Med 2017; 79:2896-2901.

34. Ligneul C, Valette J. Probing metabolite diffusion at ultra-short time scales in the mouse brain using optimized oscillating gradients and "short"-echo-time diffusion-weighted MRS. NMR Biomed 2017; 30:e3671.

35. Kan HE, Techawiboonwong A, van Osch MJP, Versluis MJ, Deelchand DK, Henry P-G, Marjańska M, van Buchem MA, Webb AG, Ronen I. Differences in apparent diffusion coefficients of brain metabolites between grey and white matter in the human brain measured at 7 T. Magn Reson Med 2012; 67:12031209.

36. Najac C, Marchadour C, Guillermier M, Houitte D, Slavov V, Brouillet E, Hantraye P, Lebon V, Valette J. Intracellular metabolites in the primate brain are primarily localized in long fibers rather than in cell bodies, as shown by diffusion-weighted magnetic resonance spectroscopy. Neuroimage 2014; 90:374380.

37. Fotso K, Dager SR, Landow A, Ackley E, Myers O, Dixon M, Shaw D, Corrigan NM, Posse S. Diffusion tensor spectroscopic imaging of the human brain in children and adults. Magn Reson Med 2017; 78:1246-1256.

38. Ellegood J, Hanstock CC, Beaulieu C. Trace apparent diffusion coefficients of metabolites in human brain using diffusion weighted magnetic resonance spectroscopy. Magn Reson Med 2005; 53:10251032.

39. Ellegood J, Hanstock CC, Beaulieu C. Considerations for measuring the fractional anisotropy of 
metabolites with diffusion tensor spectroscopy. NMR Biomed 2011; 24:270-280.

40. Harada M, Uno M, Hong F, Hisaoka S, Nishitani H, Matsuda T. Diffusion-weighted in vivo localized proton MR spectroscopy of human cerebral ischemia and tumor. NMR Biomed 2002; 15:69-74. 


\section{Figures}

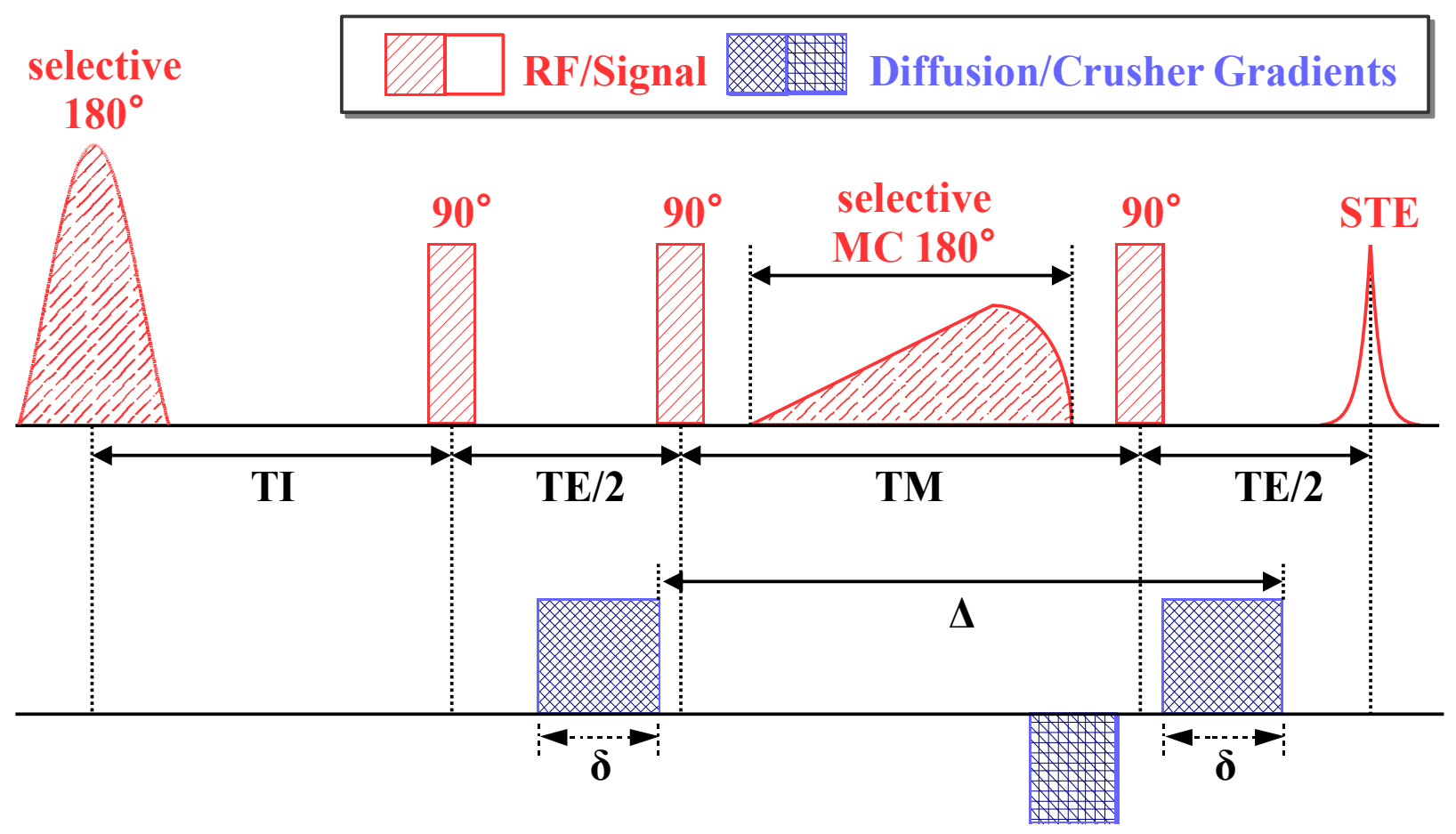

Figure 1:

Sketch of the non-water-suppressed diffusion-weighted STEAM spectroscopy sequence with metabolite-cycling (selective $180^{\circ}$ : water-selective inversion pulse; selective MC $180^{\circ}$ : metaboliteselective adiabatic metabolite-cycling pulse; STE: stimulated echo; for other abbreviations, see text). 
Raw data after PhCor, FrCor,

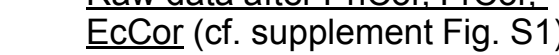

Motion Compensation (MoCom)

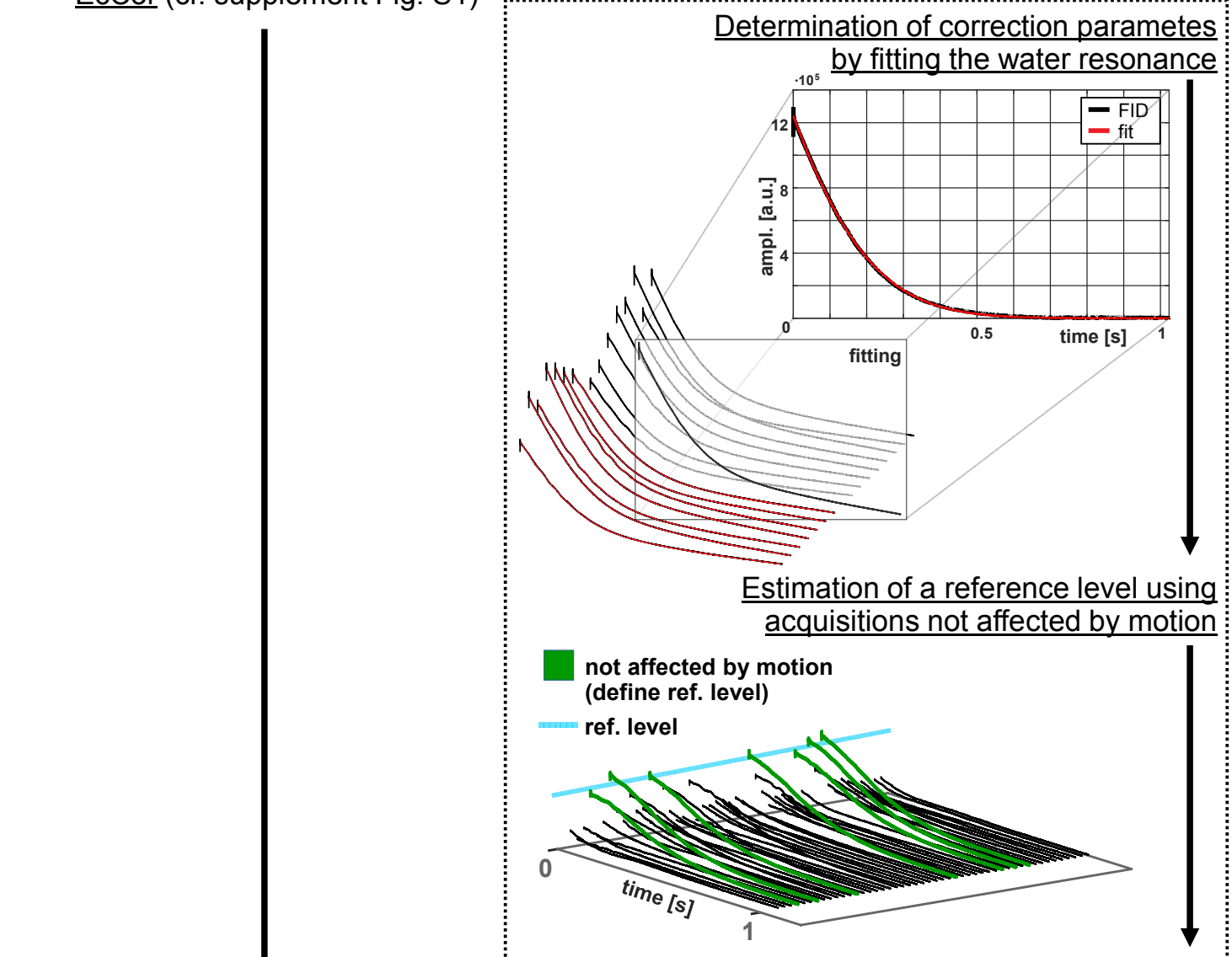

Restoration by signal rescaling and exclusion

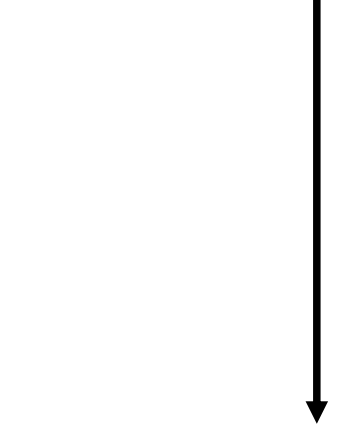

discarded FIDs

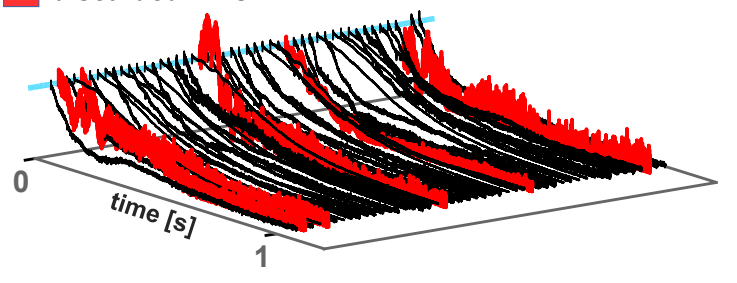

Weighted averaging to preserve SNR

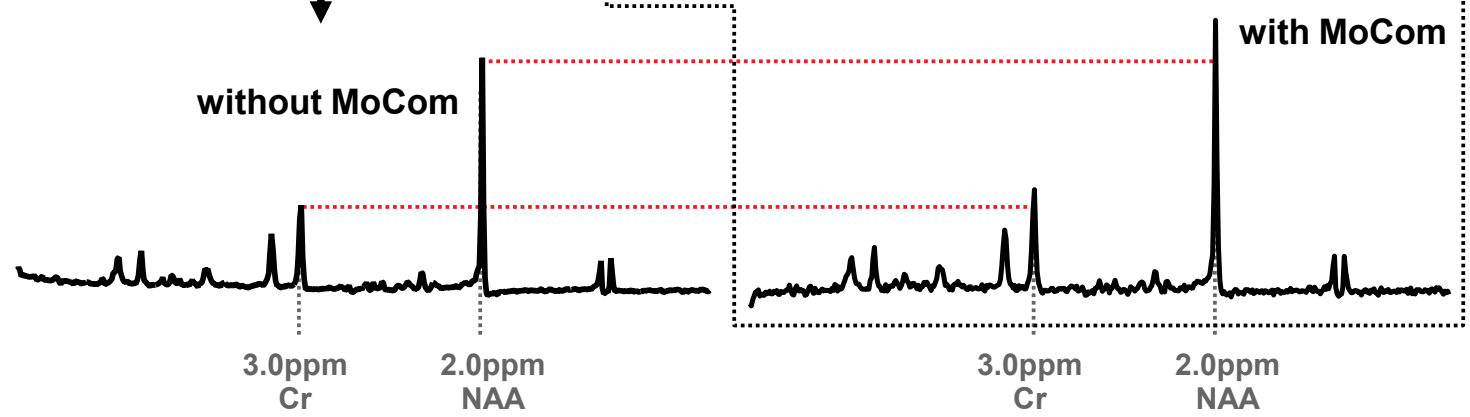

Figure 2:

Illustration of implemented MoCom scheme. After basic reference corrections for frequency- and phase-alignments as well as eddy-current correction (see text), an intensity correction is applied to all scans based on a reference level obtained from a predefined top percentile of the scans. Heavily motion affected scans are rejected and weighted averaging leads to the final restored spectrum. 

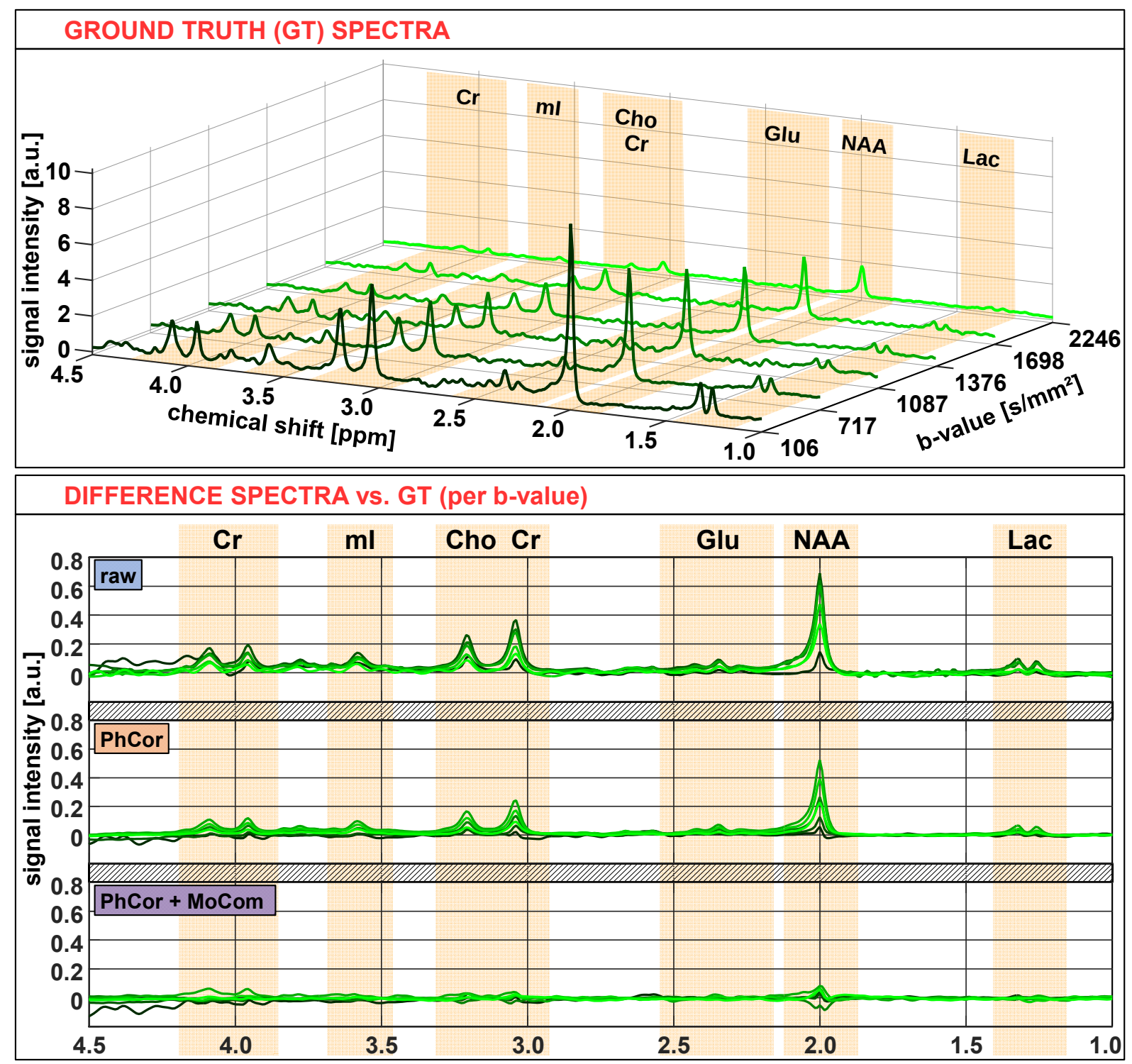

DIFFERENCE SPECTRA vs. GT (average of all b-values)

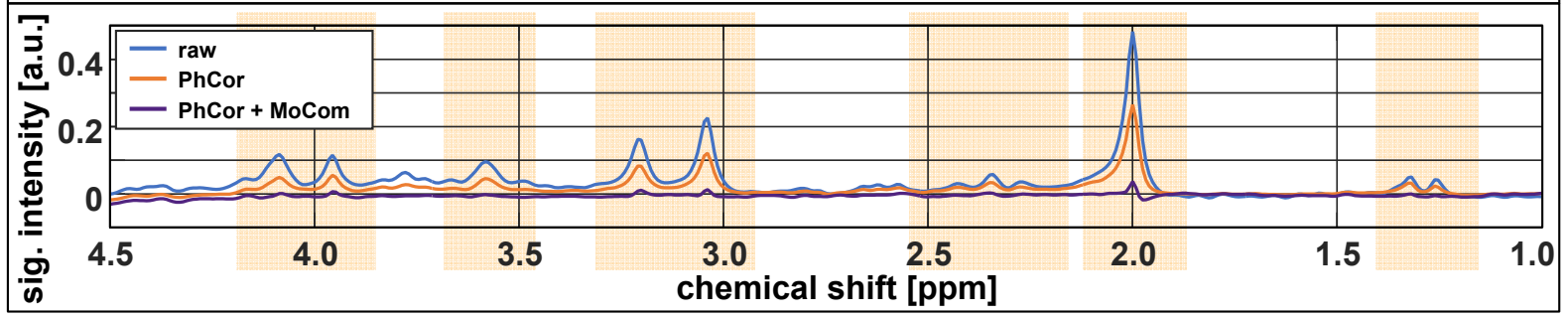

Figure 3:

Illustration of the effect of phase and motion correction steps on spectra from a rotating phantom. A) Ground Truth (GT) metabolite spectra as function of b-value for reference from the stationary phantom (PhCor applied, $1 \mathrm{~Hz}$ Gaussian apodization for visualization only). B) Difference between GT and spectra from a rotating phantom for different correction schemes. C) For better visualization of signal restoration by MoCom difference spectra averaged over all b-values are shown (MoCom with a reference level of $15 \%)$. 

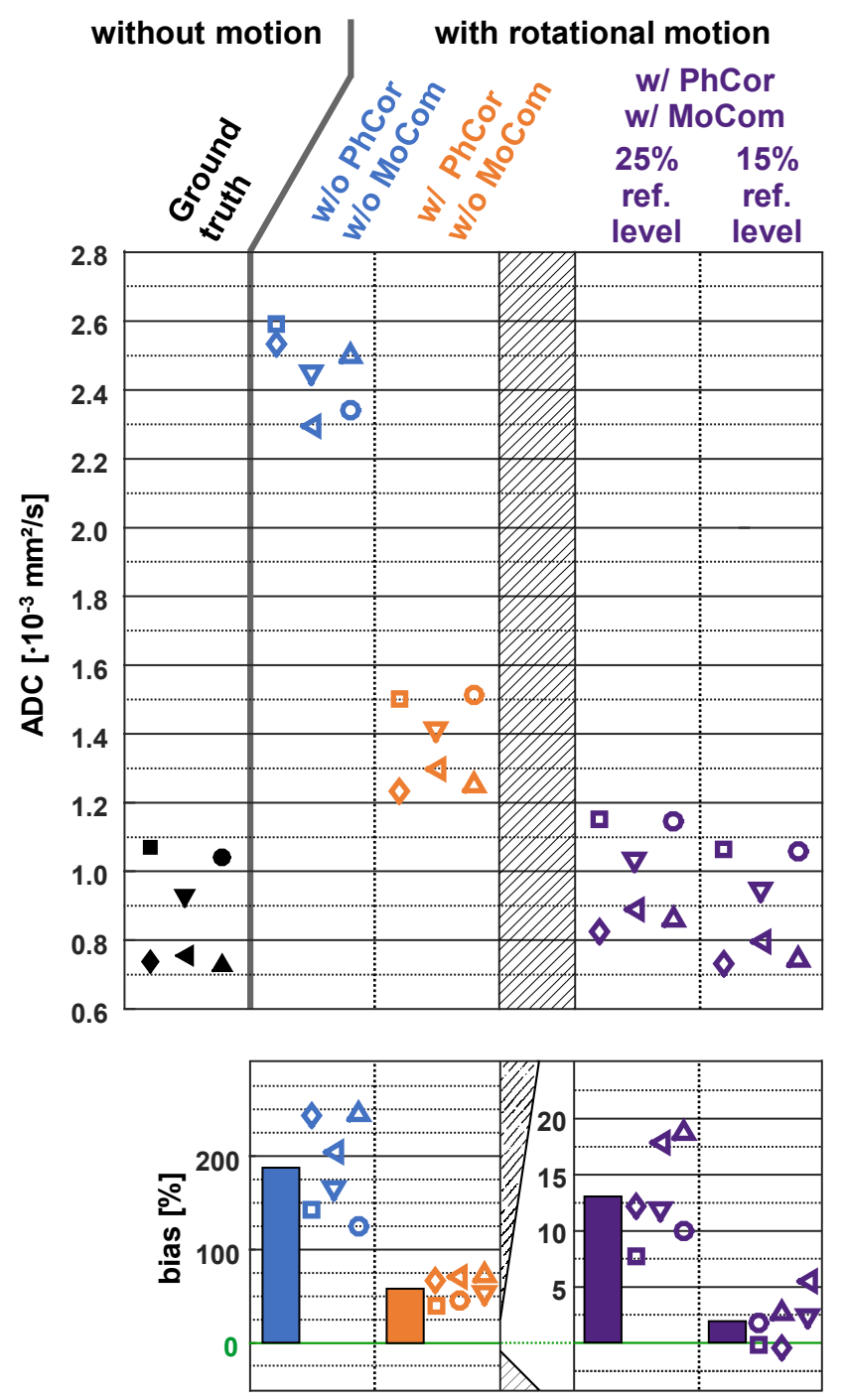

$\square$ Cho $\bullet$ Lac $\nabla \mathrm{Cr} \diamond \mathrm{Glu} \measuredangle \mathrm{NAA} \triangle \mathrm{ml}$

\section{Figure 4:}

Illustration of the effect of phase correction (PhCor) and motion compensation (MoCom) on estimated absolute metabolite ADC values for a rotating "braino" phantom (A). The bias with respect to the GT ADCs is presented in B). Without any correction, the metabolite ADCs are overestimated by $188 \%$ on average. PhCor alone reduces this deviation to $58 \%$ and restores the metabolite specific ordering of $A D C$ values. The combination of PhCor and MoCom was tested with two different reference levels. At $25 \%$ the $A D C$ overestimation is reduced to $13 \%$ on average. Using a level of $15 \%$ almost completely restores the GT ADC values. 

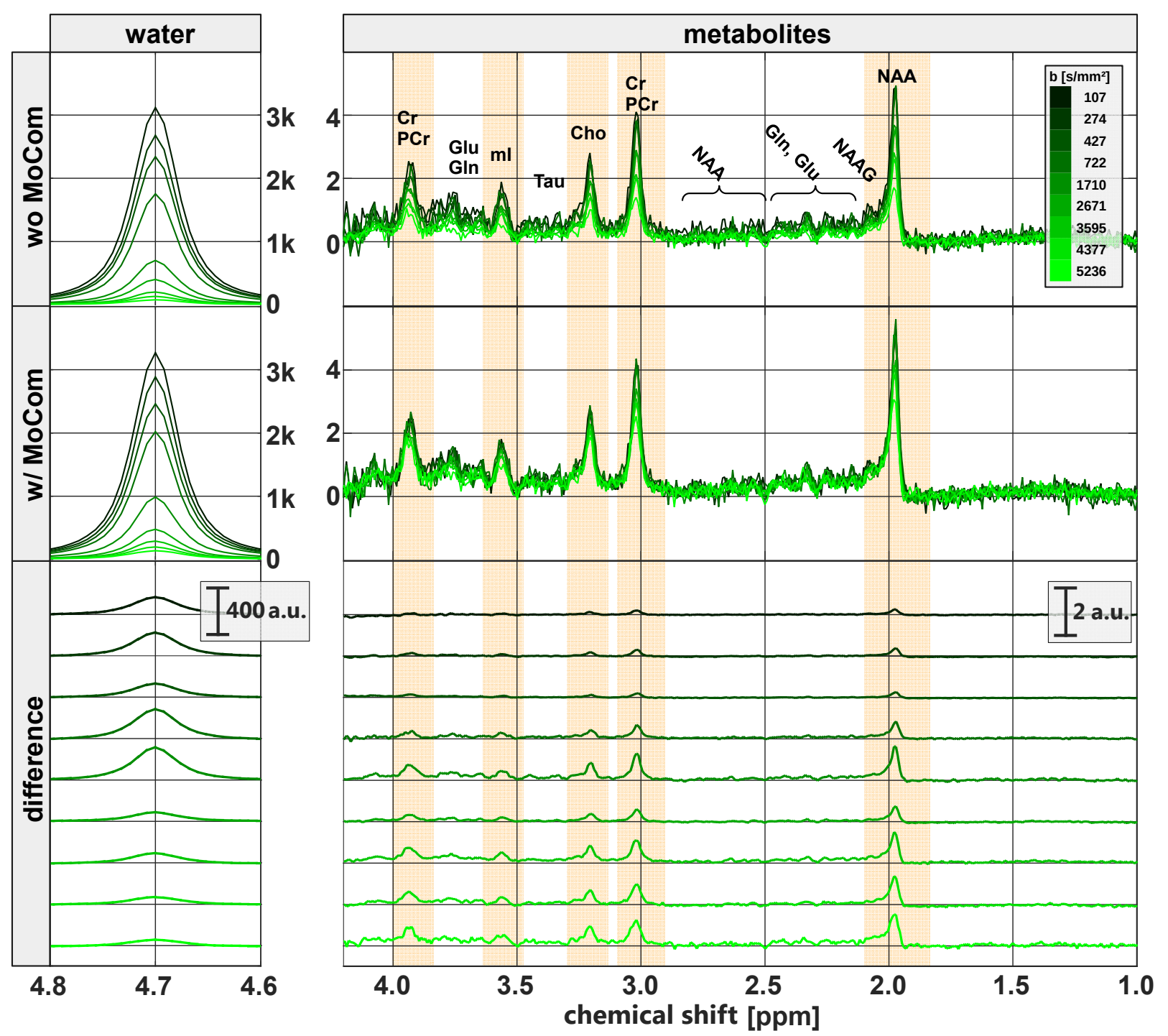

Figure 5:

Illustration of effect of motion compensation (MoCom) on spectra for a case of a single human subject (39 year old male). The resulting water and metabolite spectra with (top) and without (middle) MoCom, as well as their difference (bottom) are displayed for all b-values. The motion-related signal loss compensated by MoCom is more pronounced at higher diffusion weightings. In absolute terms, the effect does not increase further beyond $b=1710 \mathrm{~s} / \mathrm{mm}^{2}$. However, relative to the remaining DW signal at higher b-values, the MoCom effect still increases also with larger b-values. 


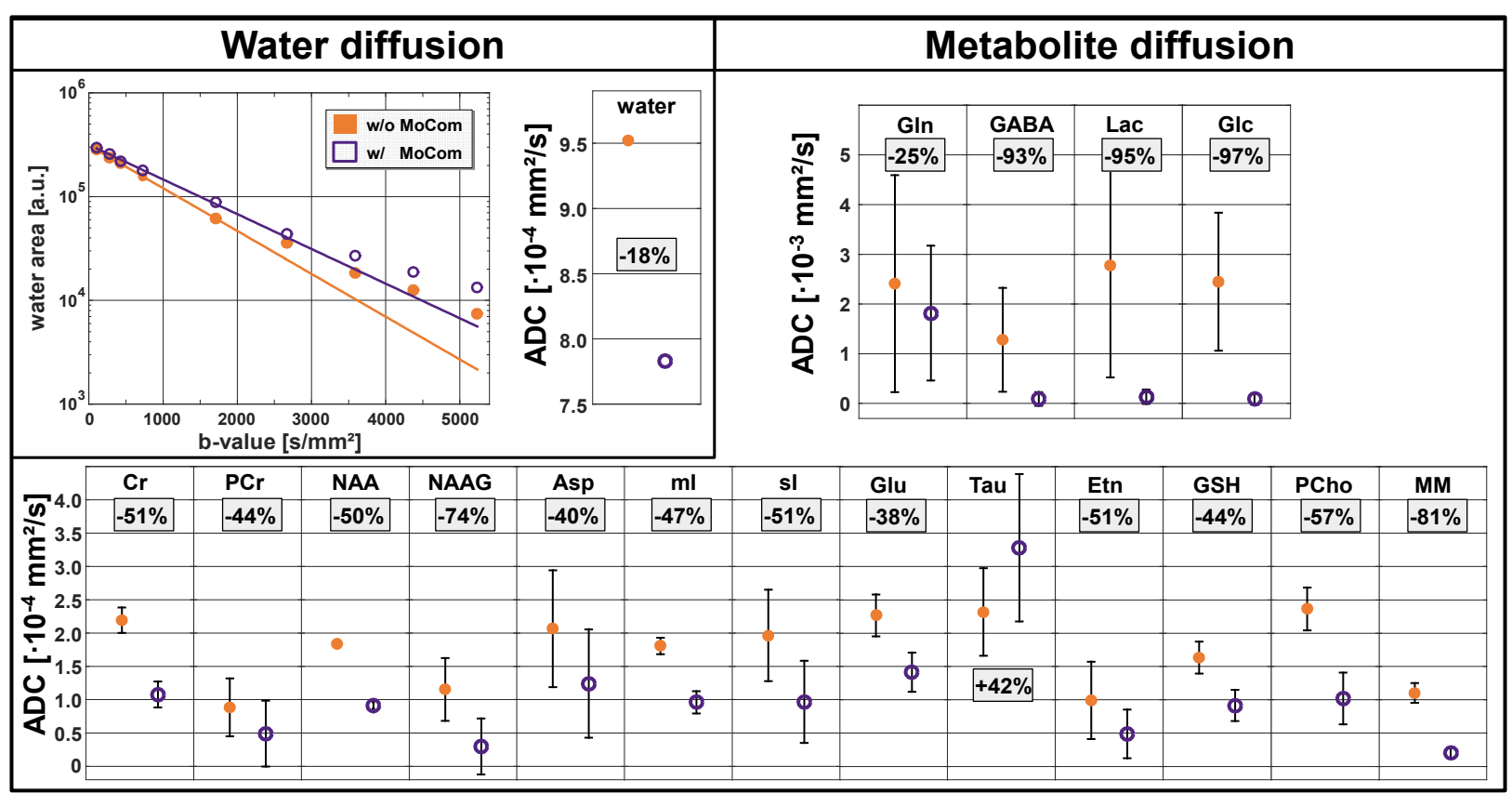

Figure 6:

Illustration of effect of motion compensation on estimated ADC values for the case of a single human subject (same case as in Figure 5, estimated ADC values printed with $\pm 1 C R L B$ as error bars, filled symbols with MoCom, open symbols without MoCom; effect size for application of MoCom indicated in $\%$ for each metabolite). A reduction in the estimated ADC value was found for water and all metabolites, except for Tau. In addition, the CRLBs for the estimated ADCs are also lower after MoCom for most metabolites. The water signal-attenuation exhibits a non-mono-exponential decay, hence the ADC for water was estimated for $b<1710 \mathrm{~s} / \mathrm{mm}^{2}$. 


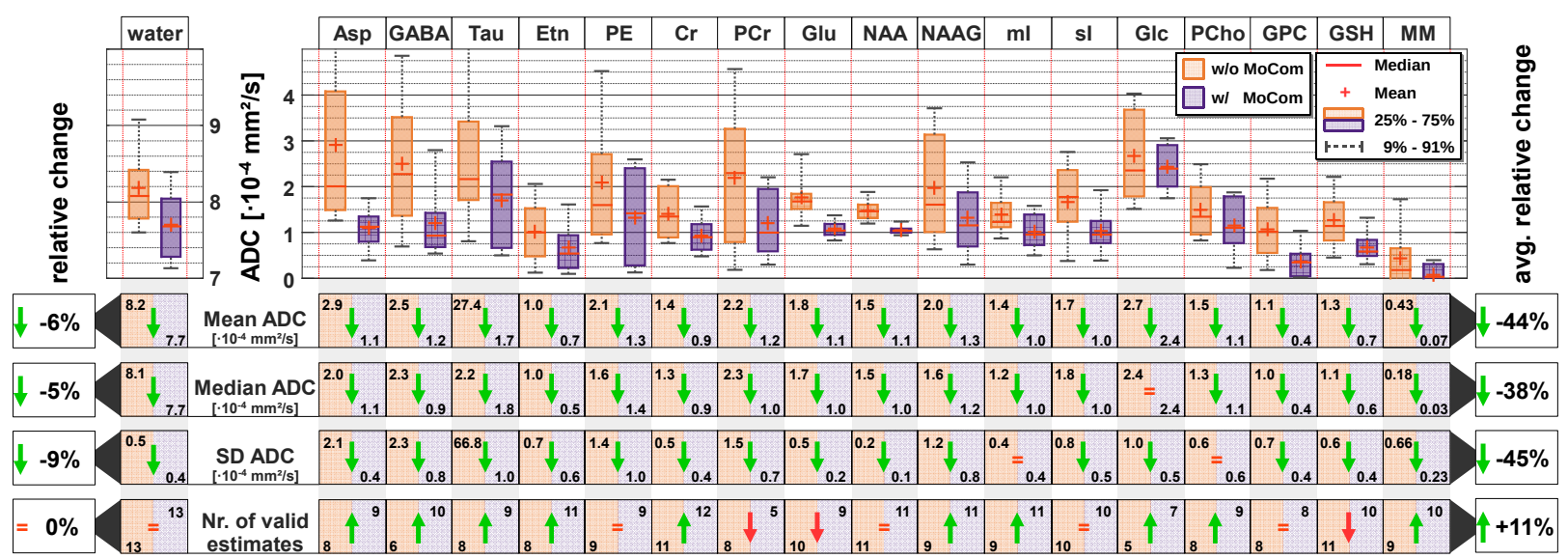

\section{Figure 7:}

Cohort results for water and metabolite ADCs obtained from 2D simultaneous fitting. They support the observations already found in the single volunteer (cf. Fig. 6). While the mean water ADC reduces only modestly by $6 \%$, the metabolite ADCs change more heavily, with a mean reduction of $44 \%$ with MoCom. In addition, the standard deviations (SD) and numbers of valid estimates improve. These results are reproduced for 1D sequential fits as presented in the supplements in Figure. S4. 


\section{Supporting information}

Supporting Figure S1:

Illustration of the established correction steps using unsuppressed water signals

Supporting Figure S2:

Illustration of effects of the different signal processing steps on ADC estimation in the stationary phantom

Supporting Figure S3:

Depiction of weighted mono-exponential fits for exemplary metabolite ADCs

\section{Supporting Figure S4:}

Tabulated cohort ADCs as obtained by sequential fitting.

\section{Supporting Figure S5:}

Visualization of definition of the reference level using the top $15^{\text {th }}$ percentile for two subjects. 